\title{
The Major Roles Of Long Distance Bus Transport In Developing Countries With Emphasis On Addis Ababa, Ethiopia
}

\begin{abstract}
This article presents the major roles of long distance bus transport that radiates from Addis Ababa to the hinterland. The purpose is to assess and identify the major roles of Long Distance Bus Transport in Addis Ababa. The methodology is focused on both primary and secondary sources. The primary informants, who were principally distinguished from the passengers, operators, and key government officials from the transport office, include the head of bus terminal and Association, and selected experts. In terms of analysis, the qualitative approach was used by applying a thick description of the issue. The findings revealed that the availability of towns comes mainly because of the routes which give access and distribution of road passenger transport. The LDB (Long Distance Bus) along the line has played a great role in the making of metropolitan linkage. The presence of these routes contributes more to the rise of urbanization and it has also played a role in the creation of towns and rural areas found around certain radius at the two sides of highways. Thus, it has a high probability for the expansion of urban sprawl and formation of conurbation in the future. The prescription for this study is to work with stakeholders to reduce the congestion of passengers in the terminals and the association also should serve the society $24 / 7$ or $18 / 7$.
\end{abstract}

Keywords: Long Distance Bus dispatch, Metropolitan linkage, hinterland, Industry

\section{Introduction}

Ethiopia is located in the Horn of Africa with the area coverage approximately $1,221,900$ square kilometers; it is nearly the size of France, Germany and the United Kingdom combined (AACC, 2009). Ethiopia is also geographically located $3^{\circ}-15^{\circ} \mathrm{N}$ and $33^{\circ}-48^{\circ} \mathrm{E}$ (ERA, 2005 and CSA, 2007). The elevation ranges from 1,500 to 3,000 meters above sea level. The World Bank (2008) indicates that Ethiopia had a population of 76.5 million in 2007; the existing population of Ethiopia is more than 80 million, which makes the nation the second most populous in Africa, after Nigeria. And the population projection 
for the future is that it will reach 106 million by 2020 and 180 million by 2050 (Oladele, 2010).

The physical set up of Addis Ababa is found at the heart of the nation and is located at $9^{\circ} 02^{\prime} \mathrm{N}$ to $9.03^{\circ} \mathrm{N} 38^{\circ} 44^{\prime} \mathrm{E}$ to $38.74^{\circ} \mathrm{E}$ (ERA, 2005 and CSA, 2007). Addis Ababa is the capital city of Ethiopia and the African Union. Again, it is the largest city in Ethiopia, with a population of $3,384,569$ according to the 2007 population census (CSA, 2007). And various estimates also show that by 2020 the city is expected to host about 6-7 million inhabitants (Iginis, 2008 as cited in Mesfine, 2009). The city of Addis Ababa has the dual status of both a city and a state capital (CSA, 2007 and MoFED, 2006) (Map 1).

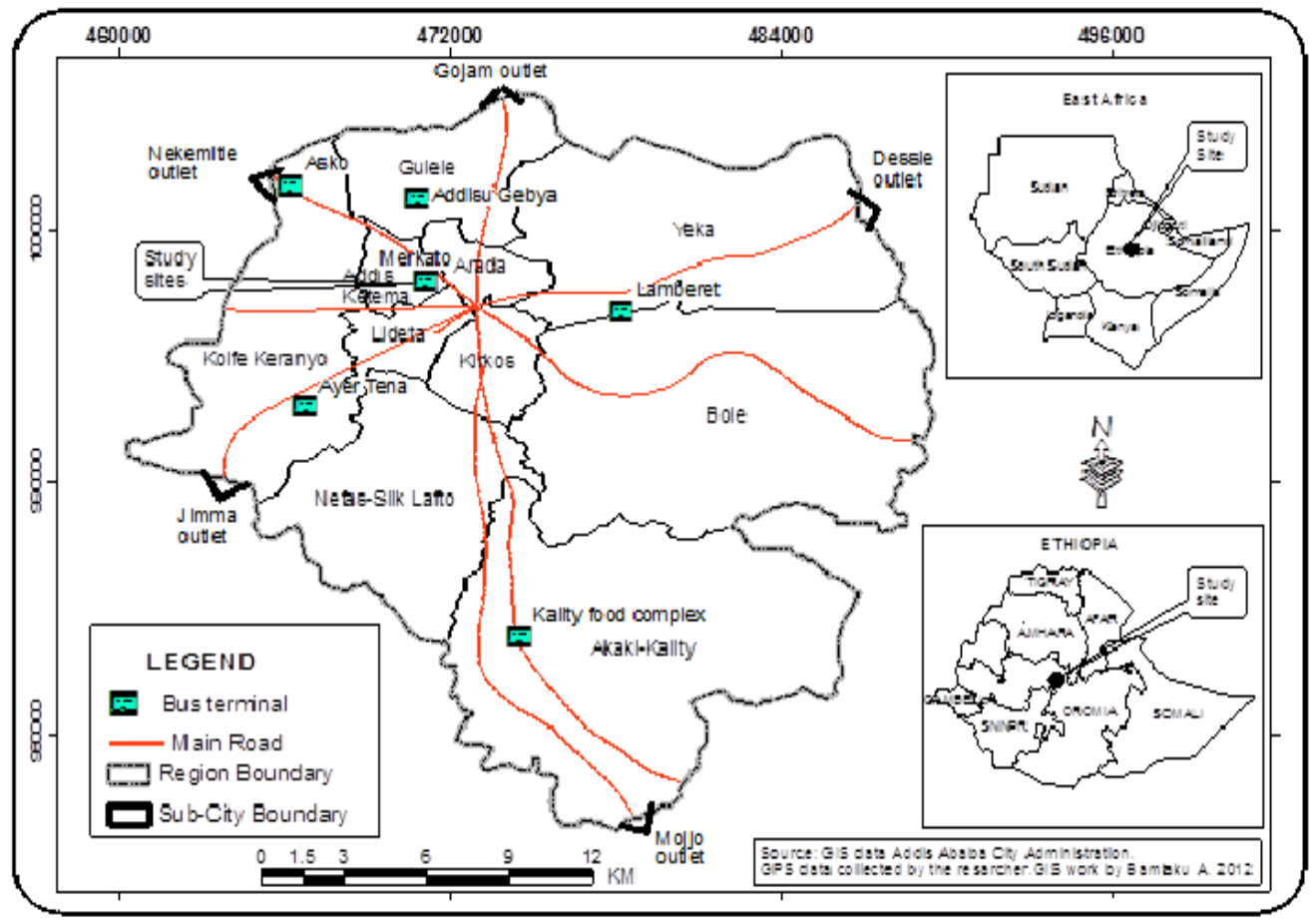

Map 1: the geographic placement of Ethiopia and the capital Addis Ababa

Transport has an important role to play in the economic growth and social development of Ethiopia. Land transportation in general, among other modes, and road transport in particular is the most widely used transport sector all over the world. It also provides a base for local, national, regional and international flow of goods and passengers. Asnake (2006) states that road transport sector plays a significant role in the national economy of developing countries through direct contribution to GDP and employment. Indirectly it 
also provides the services that are indispensable for the development of other economic sectors. Road transportation plays a vital role in the distribution of essential goods and services from place to place (Meket, 1997; Herbert, 1979 as cited in Yayeh, 2003).

In Africa, road transport is the dominant mode of motorized transport that accounts for $80 \%$ of the goods traffic and $90 \%$ of the passenger traffic in the continent. There are three modal systems of transport that exist in the country (road, air and rail). Nonetheless, studies that were conducted in the country, ERA (2005) and EFTA (2011), note that about $99.31 \%$ of all passengers use road transport for their mobility, $0.65 \%$ use airline and $0.04 \%$ use railway transport. This indicates that the mobility of the society is highly dependent on road transport industry rather than other modes (ERA, 2005). And the Ethiopian Federal Transport Authority EFTA (2011) report that the sector is facing certain challenges because the entire population of the nation rely on road transport much more than on other alternative modes. There is also prevalence of poor quality services in the sector. As a result of this report, it can be noted that the existing road passenger transportation of the nation is not satisfactory. This implies that it is important to maximize service satisfaction in the industry.

The EFTA (2011) report indicates that there is a slight growth in the passenger transport industry, particularly in the medium commercial passenger transport (about 15.7\%). The maximum growth $(74.7 \%$ ) is registered in the small commercial passenger road transport sector such as the minibuses, while long distance buses show a slight increase $(9.6 \%)$. The report further indicates that in 2010, the total number of passenger transport vehicles that rigorously served the society was 13,684 . Of these, about $7.75 \%$ were long distance buses with 44 and more seats and the rest 12,623 (about 92.25\%) were buses with 24-44 seats. This indicates that the growth of cross-country commercial passenger transport is relatively small in terms of quantity. With regard to the number and types of buses by levels, in 2010 only 23 buses worked as level one, 381 as level two and 657 as level three. The level three comprises only about $61.9 \%$ of all buses in the nation (EFTA, 2011). This puts the adequacy of the service provision into question.

Again, with regard to the passengers transported, the CSA (2009) gives a report of passenger and freight transport activities in the nation. It indicates that from 2004 to 2008, the growth rate of transported passengers by medium and large passenger road transport has risen from $4.1 \%$ to $6.7 \%$ (SBPLC, 2009). In terms of vehicle spread in the nation, Addis Ababa holds more than $75 \%$. Again, out of the total number of vehicles in the country, which is about 400,000, about 130,000 vehicles in 2004 were located in Addis Ababa. Passenger road transport comprises about $45 \%$ of the total vehicle stock in the city (EFTA, 2011). Again, from the total long distance bus transport of the nation, about $90 \%$ are found in the city. This is why the 
investigator intended to make this study in the city. This research focuses on passenger road transport mainly in Addis Ababa, the national capital. It also examines the roles of transport in linking the capital with other cities and towns.

Existing studies on transport, which have been carried out in the nation, do not properly address the issue under investigation. For instance, ERA (2005) designed the national urban transport policy that examined the general situation of road network rather than the inter-urban passenger transport. Mintesnot and Takano (2007) made a diagnostic evaluation of public transportation mode choice in Addis Ababa with a special focus on intra-urban government bus transport. And AACC made a study in 2009 on the management of commercial road transport in Ethiopia. Among these and other studies, no one made a study on the major roles of long distance buses in Addis Ababa. This indicates that there is a gap in the studies with regards to the role of the sector. Therefore, this study is intended to fill that gap and investigate the major roles of long distance buses in Addis Ababa in Ethiopia.

\section{Objectives and methodology}

The focus of this study is to assess the major roles of long distance bus transport in Addis Ababa, Ethiopia. Therefore, as per the insight of this objective the following methodological mechanism was undertaken. In light of research design, it employed qualitative and quantitative approaches. Both qualitative and quantitative approaches were chosen for the analysis, but strong emphasis is given to qualitative and less weight for quantitative approach. The philosophical views of the study are focusing more on the positivist view that deals with quantitative mechanism while the phenomenological one is focused on the interpretative way. Both primary and secondary data sources were used for this study. The primary sources were obtained via interview, structured interview (questionnaire), observation tools and focus group discussions. The additional data also collected by the instruments used for this study are maps, photographs and other.

On the subject of place and study site selection, it was carried out in the city of Addis Ababa, especially in Mercato terminal (map 1). It has been the main and the only national bus terminal that serves the whole nation and it is also placed at the center. And also, it has high accumulation of formal LDB operators who are working from this terminal. Routes and towns for the survey were selected by using the lottery method. Out of 5 major highway routes that link the city with hinterland, four were selected by the lottery method. These 4 lines and towns which were selected for this survey are Metehara, Debre Sina, Gebre Guracha and Hosanna. And the key government officials 
are those who are currently working on the formal LDB such as heads of bus terminal, head of Associations, and traffic officers of the city.

In this way, selected types of informants are distinguished; principally about 241 informants were taken from the passengers of LDB. The researcher selected about $10 \%$ of the passengers from each bus and these individuals were asked to fill the questionnaire, and about 6 passengers from each sampled bus multiplied by 8 buses and again multiplied by four routes at two survey seasons (i.e. $6 * 8 * 4 * 2=384$ ). In total, 384 passengers were surveyed. Out of this, only about 241 questionnaires were fully completed and returned. The passengers' selection was also made by using a systematic approach that took the $\mathrm{K}^{\text {th }}$ bus alongside the sampled routes.

With regard to the operators, there were about 64 buses or operators selected by systematic sampling. In order to know the total number of operators, it is necessary to know the amount of buses that are dispatched from the terminal. It was found that there are about 100 to 120 large buses which leave the terminal, and a similar number also enters the terminal every day. Out of these, $6 \%$ of the buses from each route took about 8 buses which were selected from both dispatching and returning buses along 4 routes at two survey seasons (i.e. $8 * 4 * 2=64$ ). Therefore, the researcher took 64 drivers/operators for this study. The required information for the selection of buses was plate numbers, side reference numbers, name of Association and so on.

The questionnaire was used as the main data gathering instrument. It incorporates about 87 questions for operators and 70 questions for passengers. The questions were primarily focused on major roles of the study. The data from passengers and operators was gathered by longitudinal survey method four times at two off and on journey seasons. It took place in 2011 and 2012; particularly it was from 1 September up to 15 January (on-season) in 2011, and April to May 2012 (off-season). Observation was guided by a check list and mainly focused on the roles of the sector; it took mostly early and late evenings for some consecutive days. The other informants are particularly the off-journey passengers at their loading place or in the terminal where they participated in Focus group discussions (FGDs), which were comprised of 4 to 5 participants. The analysis is also made based on some quantitative methods like the inferential statistics and the high emphasis is also given to qualitative and content analysis methods which were undertaken to analyze the open ended questionnaire and informal oral and structured interview. It was primarily carried out by using thick description of the issue.

\section{Results and discussion}

This chapter presents the major roles of long distance bus (LDB) transport and its contribution to the formation of metropolitan linkage 
of Addis Ababa with hinterlands. The roles that the industry performs in the society can be seen in light of both direct roles and indirect roles, and the general and specific roles. The dispatch of LDB has a significant role in the linkage of the city with other major towns and contributes a lot to the metropolitan linkage of the nation. It is found that it has notable roles at the origin, transition and destination places, and on the other parts of the nation. Hereunder follows a detailed discussion on the roles of the sector.

\section{Main outlets and their roles in metropolitan linkage}

The study has found that the linkage can be seen from different perspectives such as market, goods and services, production and physical linkage. Physical linkage can be seen in terms of roads that run within different places. One of the pillar mechanisms for creating the linkage among various towns is the presence of highway roads that can create a physical link between towns and surrounding rural areas. This link by itself has prominent roles to play as supplementary links to market, goods and services, societal, cultural and the likes because it is commonly carried out by using large distance buses and would also contribute to metropolitan linkage of a city with hinterland. The concept of metropolitan linkage is understood as a link that connects the hubs with the overwhelming part of the nation. In other words, it is a kind of link which connects the metropolis with the hinterland.

With regard to the nature of metropolitan linkage, there are at least five major outlets (or highway routes) that can link Addis Ababa with the hinterland (Map 1, 2 and 3). They include Gojam, Nekemte, Jimma, Mojo and Dessie outlets that interlink the city with all outward and inward directions of the nation. The Mojo outlet has a route which is located on the south and east lines and connects Addis Ababa with major towns located in the southeast direction. It is also the line of Ethio-Djibouti and Ethio-Kenya corridor that links Somalia, Kenya and Djibouti. Due to this, there is a large flow of buses which interlink the nation via the Mojo outlet. The Dessie route links the northwestern part of Ethiopia with Ethio-Eritrea and Sudan boundary. In this survey, this outlet is the second busiest, which gets LDB transport, next to Mojo. In contrast, other outlets cover only a small figure. The Gojam and Nekemte lines link the western and northwestern part of the nation and finally they also interlink Sudan and South Sudan. The Jimma route leads to the southwestern part of the nation and finally it can make a link with Kenya. Again, the other outlets like Jimma, Gojam and Nekemte get the lowest flow of LDB (Map 1). 


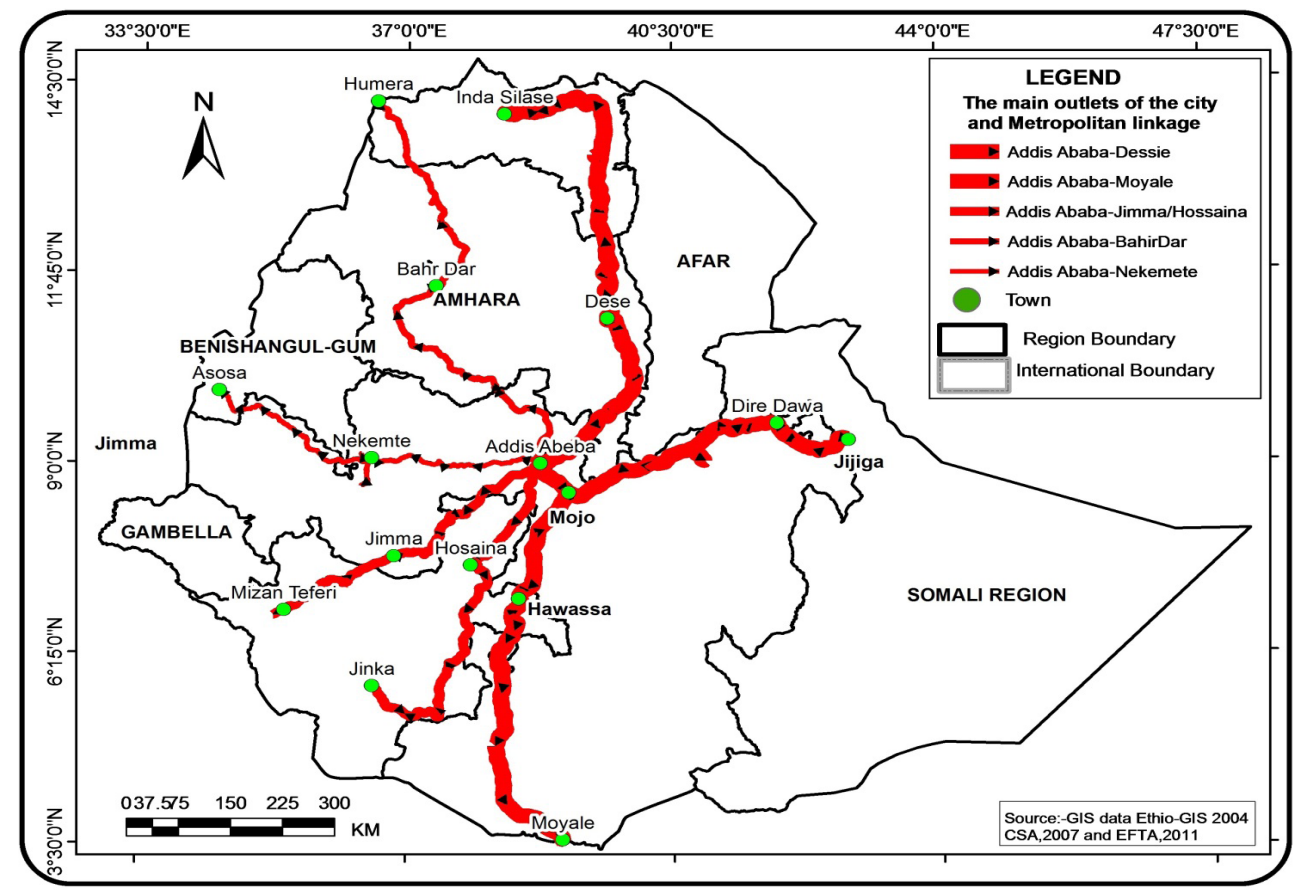

Map 1: The main outlets of the city and the general flow of buses per outlets Source: Adopted from Google maps (Google software)

The role of LDB for Metropolis and Metropolitan Linkage is very obvious. It has a major role in the integration and growth of cities and towns that are located along the flow lines of buses. Even the highway roads attract the small settlement from rural villages that are settled along the route and finally they can form a town. This implies that the infrastructural facility is a base for the settlement and contributes to the formation of the town. Thus, these routes contribute to the rise of urbanization and they have a few roles in the making of towns and rural areas found around the certain radius at the two sides of the highway roads. It also indirectly connects the town and rural areas that are found on the two sides of the main routes. Commonly, the city is located within 10 to $20 \mathrm{~km}$ and more radius of the town along the route. And it has also brought the fact that LDB primarily links the city with other parts of the nation. Thus, the LDB has contributed much to metropolitan linkage. 

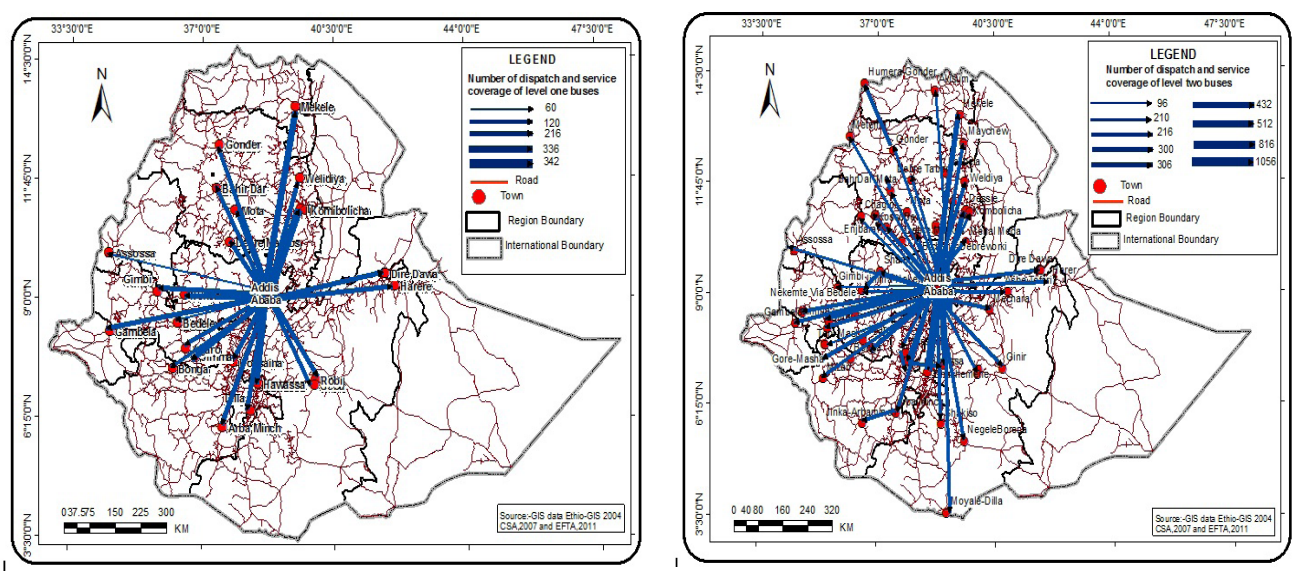

Map 2: Half year dispatch of level one and two buses per towns
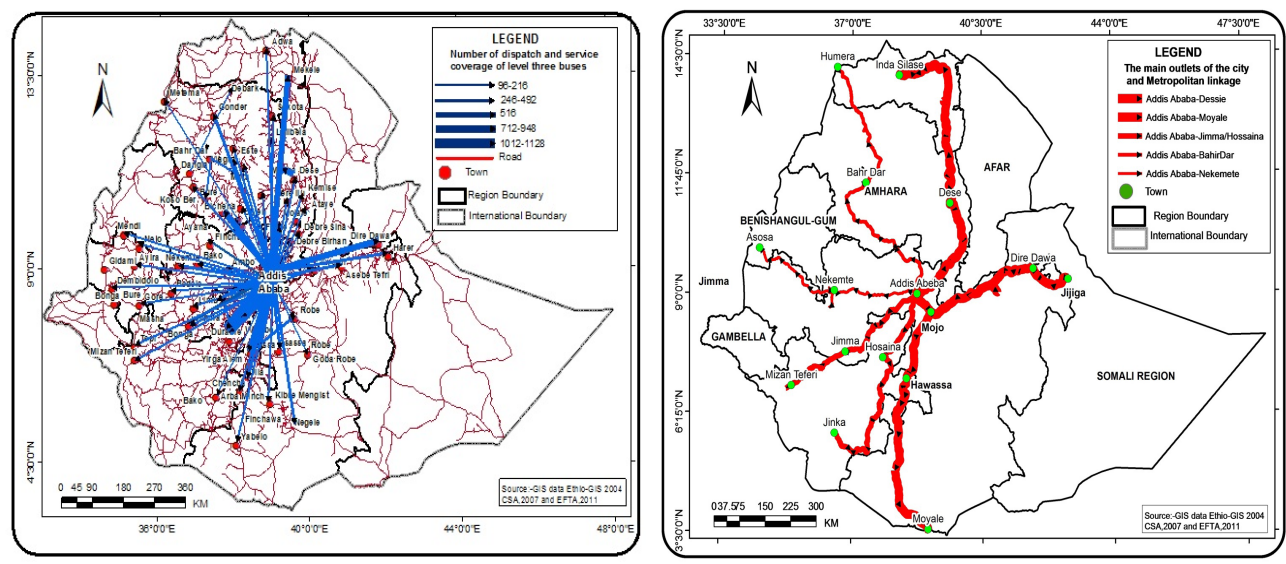

Map 3: Half year dispatch of level three, and the general flow of buses per outlets

The actual and potential factors for the outgoing and incoming mobility of buses along the paths lead towards and out of Addis Ababa. The maps 1 to 3 explain that all routes that are coming and going towards and out of Addis Ababa serve as radial links to the surrounding major towns. In comparison, ERA (2005) documents discuss that the entire road system of the nation is constructed by using Addis Ababa as a springboard. It is important for the interwoven nature of all towns and surrounding rural areas that are located at nearby places of the city. It has also shown a radial linkage of the city and hinterland. This radial linkage of the city can be expressed with the old saying.

The reality of the city exhibits similar feature to ex-Rome. As the old saying 
goes, "all routes lead to Rome". It was stated during the time of the former Roman Empire when it was strong and Rome was an important figure in the world. And just as to its intended theme, this proverb apparently was used in the medieval times when all roads in the Roman Empire literally led to the capital city of Rome as the economic and political dominion of the empire. It is simply denoted that all the existing paths that are found in the nation are linked and lead to the same destination, Addis Ababa. The finding reflects that the city of Addis Ababa plays a similar role to that of the former Rome. It has been relatively highly interlinked with all major routes of the nation. This shows that it has provided a radial service to all outlets of the nation. This physical linkage of the routes by itself contributes a lot to the mobility of the society, which is achieved by the help of long distance buses.

Addis Ababa is an area that became a metropolis and an area of attention for getting radial linkage, and this is mainly because of the geographic placement of the city which is located in the center, and it is the first modern headquarters of the nation which has served as a city for long periods as of 1896. And also it has a high concentration of service sectors. The other reality of the city is the saturated and concentrated area of most industries. Moreover, it is an area of the concentration of international offices and embassies as well. According to AACGO (2011), CSA (2007), and ERA (2005), it is the diplomatic capital of Africa; the fact that it is home for over 100 embassies, and diplomatic missions are located in the capital makes Addis Ababa one of the important international cities and the center of tourism and conferences. This explains all the international mobility carried out from and into Addis Ababa.

Almost all Federal bureaus are concentrated in it and it is an area that has a concentration of NGOs and where many national meetings are organized. The city is found to be in a relatively advanced position compared to other towns or cities in the nation. Owing to this, the government, NGOs, self-employed individuals and other bodies likely prefer a job transfer in the city, and even the new job-seekers also compete in order to get a job and live there. Finally, it is also a center of political, economic, geographic and social capitals of the nation. All these features contributed a lot to the city becoming an area for the outlets and inlets of the nation. Thus, all the geographic, political, economic and social aspects of the city would become more advantageous and contribute a lot to the metropolitan linkage with the hinterland.

Addis Ababa can be accessed with all levels of long distance buses and has a stronger linkage than other towns because it has a direct link with the hinterlands. It also indirectly connects the towns and rural areas that are found at the two sides of the main routes. Due to this, various towns and rural areas, interlinked with the major and medium towns, have a chance to make linkage. These places can be reached by altering to the other buses or other modes of transport and this is commonly carried out by using two or three towns. Thus, most of the rural villages and various small towns 
that are not located along the highway route are also indirectly interwoven with each other and benefit more from the industry. In general, the city is found in the geographic epicenter of the nation and its infrastructural and political advantage also makes the city get huge flow of buses and connect to the hinterland. This infrastructural facility, mainly roads, contributes to the creation of high urban sprawl along the routes and allows for conurbation at the nearby outlets of the city.

\section{Bus dispatch of LDB and its contribution to urbanization}

In this section it is explained how the amount and flow of bus dispatch also contribute to urbanization and vice versa. The study indicates the estimated number of towns and distance gap between both major and all towns in kilometers. The nature of the towns is determined by the size of population, socio-economic and technological aspects. And also, the dispatch of buses is also determined by various factors.

In Ethiopia, this study shows that large towns are those which have the population of more than 2 thousand, and usually are larger than a town but smaller than a city (CSA, 2007). Whereas, the world standard of major towns contains more than 5 million people (Barney Cohen, 2003). MUDC, Ministry of Urban Development and Construction report of 2007 classified the urban areas of the nation into four parts based on demographic criteria, but the socio-economic and technological aspects are not included. These are cities, large, medium and small towns. Small towns also have the population that ranges from 2,000 to 10,000 . They comprise more than 730 towns or about $89 \%$ of the total number of towns found in the nation (925). And the rest $11 \%$ represent medium, large towns and cities. This indicates that the transformation of rural areas into urban is very fast. The medium and large towns' population lies between 10,000 to 50,000 and 50,000 to 100,000 respectively. There are about 70 medium towns and about 15 to 20 large towns (MUDC, 2007). The number of cities in the nation is only 10 cities except Addis Ababa. In aggregate, the nation has about 925 towns.

Map 1 shows that the highest dispatch of long distance buses is seen along Mojo and Dessie lines. It indicates that out of these outlets, the greatest frequency of major towns was observed at Dessie or Inde Silasie routes that registered 27. It shows relatively busy flow recorded along Dessie line. It also has various small towns (78) along the route. This implies it has a higher interaction with the center than others. While the lowest distance gap between the major towns below the average $(45 \mathrm{~km})$ was realized on Jijiga $(30 \mathrm{~km})$, Inda Sillasie $(40 \mathrm{~km})$ and Bonga $(41 \mathrm{~km})$. Again, this also implies it has lower interaction with the center than others.

However, an extraordinary record is also registered in Addis Ababa. It holds 
more than 2.7 million people and is consider a mega city in the context of Ethiopia and metropolis (MUDC, 2007). It discloses that on average, there are more than 15 major towns that have made direct link and get the service of LDB from Addis Ababa. Again, with regard to the number of total towns along the main outlets, it was about 42 (figure 1 and 2). All these towns that are found along the highway benefit by receiving both direct and indirect services of long distance buses.

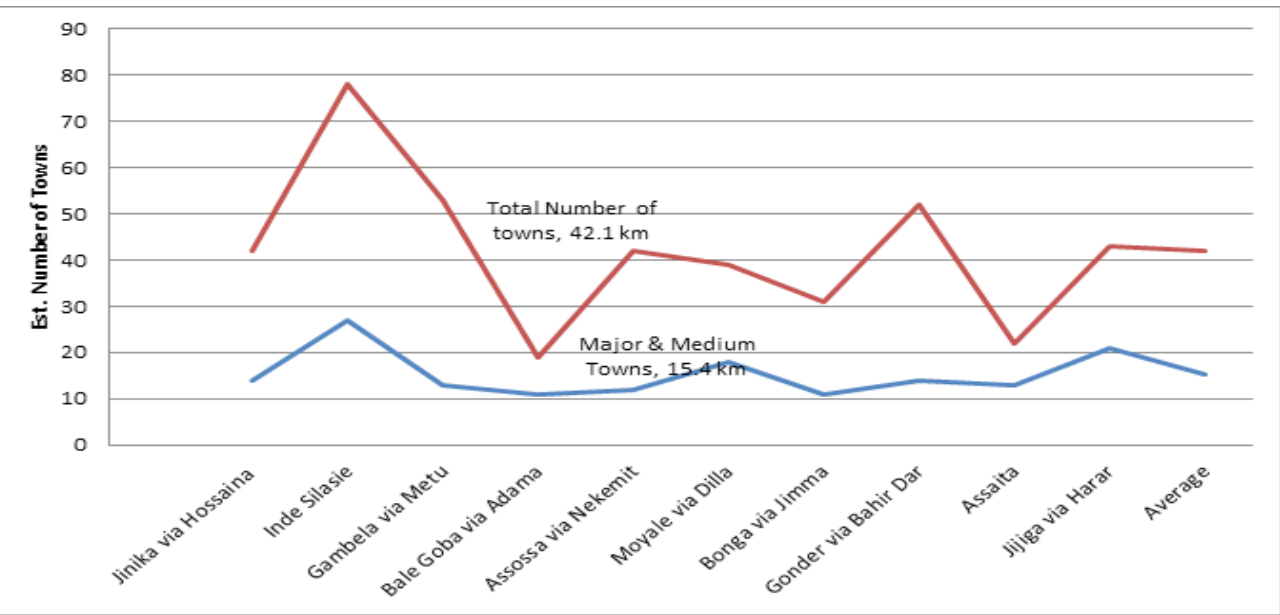

Fig 1: Estimated number of Towns along Major Routes from (O-D) Source: Compiled from GIS software and MOTC data of Jan 2012

Notice: The number of towns is manually counted by the help of EFTA data.

The survey shows that a very large number of towns is found along Inde Silassie (about 78 towns). This high presence of towns appears due to historic set up of the line that makes the suitable conditions for the formation of towns. It is also because of the very lengthy nature of the route, about $1097 \mathrm{~km}$. And it also has strong ties with the center because of the prevalence of economic and social aspects. This also implies that these areas highly benefit from the industry and the linkage is very strong compared to other outlets (Figure and Map 1). But the routes towards Gambela (53) and Gonder (52) are also more benefited than the rest of the routes. This suggests that there is a relatively high interaction compared to the other corner of the nation.

In contrast, it shows that the lowest number of major towns is found along Bonga, Bale Goba, Asosa, Assaita and Gambela routes: about 11, 11, 12, 13 and 13 towns respectively. In these routes, most of the towns are small in size that is found in the countryside owing to regular market link and act as 
business centers for surrounding farms and villages. This low number of towns appears likely because of geographic unsuitability (climate, landscape and other) of the route for the setup of towns, desertedness of the place, lowness of trade linkage and less population pressure than in other lines.

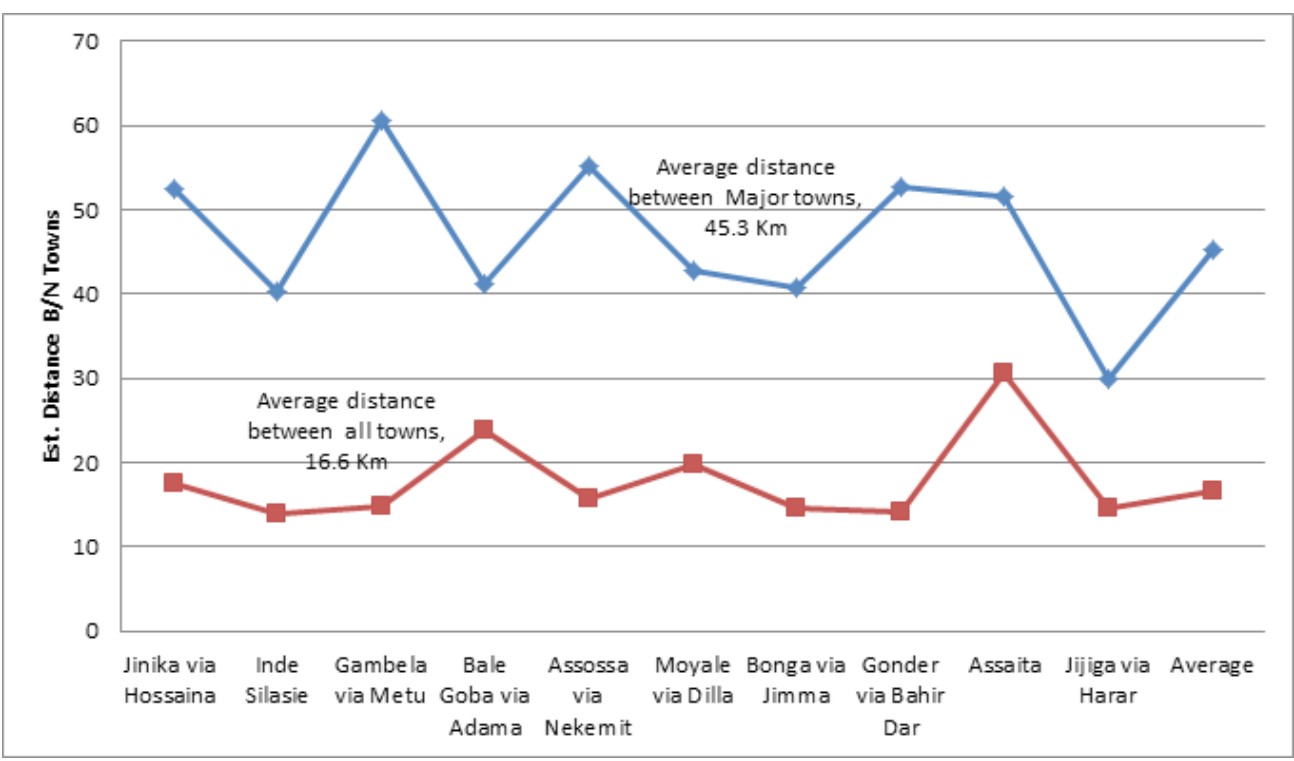

Fig 2: Average Distance Between major and all towns

Source: Compiled from GIS software and MOTC data of Jan 2012

Notice: The distance gap between towns is manually counted by the help of GIS data, EFTA data and CSA 2013 estimate.

With reference to the average distance found between the major towns, they are placed at about $45 \mathrm{~km}$ distance intervals. This shows that throughout these outlets that begin from the origin to destination, major towns would be located at $45 \mathrm{~km}$ intervals. It is due to various factors like the route being newly constructed, the economic and social ties of the community along the route being also too low and other factors. This shows that both the level and rate of urbanization along the lines of the nation is not going well (fig 2). Thus, the increment of the gap between towns results in the reduction of physical linkage of the city.

Despite the presence of extraordinary distance gaps between the major towns, it has been seen that in the selected places the distance is very large. For instance, along Gambela $(61 \mathrm{~km})$, Assossa $(55 \mathrm{~km})$, Gonder $(53 \mathrm{~km})$, Jinika $(52 \mathrm{~km})$, and Assaita $(52 \mathrm{~km})$ routes. Again, this largeness of distance between major towns along these routes has its reasons: areas which were predominantly settled in scattered pattern and have huge rural areas, and 
other special cases have emerged. While the smallest distance gap between major towns which was below the average $(45 \mathrm{~km})$ is found mainly on Jijiga $(30 \mathrm{~km})$, Inda Sillasie $(40 \mathrm{~km})$ and Bonga $(41 \mathrm{~km})$. However, in advanced countries, the gap between cities is very small and in some cases two cities become very much interconnected with each other. This growing and joining together of various towns along the route is suitable to make conurbation in the near future.

The average distance between all towns is $17 \mathrm{~km}$. Irrespective of this, the smallest distance between all towns that was recorded below the average values $(17 \mathrm{~km})$ may be studied. Out of these, the lowest distance interval has been seen on the main routes along Inda Sillasie $(14 \mathrm{~km})$, Gonder $(14.2 \mathrm{~km})$, Bonga $(14.5 \mathrm{~km})$, and Jinika $(14.6 \mathrm{~km})$. This shows that there is a tendency to create conurbation and also it contributes more to the spread of urban sprawl between towns that would appear in the long run. Thomas and LaVerle (1991) and CSA (2007) also argue that the rate and level of urbanization in Ethiopia will be accelerated. On average, the rate of urbanization spread is $6 \%$. The total number of towns in the nation has reached 925 in 2007 and rises yearly. In 1984, there were 312 urban centers with a population of more than 2000. By 1994 this number had increased to 534 and currently there are 925 urban centers showing a rapid change of population distribution in the nation. Therefore, the evidence indicates that the LDB transport might also contribute a lot to the rise of urbanization.

Finally, to estimate the accurate number of towns for the future, in 2020 it should reach 1800. This figure is calculated by taking the 1984, 1994 and 2007 census data i.e. 312 , 534, and 924 towns respectively. The numerical growth of towns in 1994 census is about more than double of 1984 and the 2007 is also about more than double of 1994, i.e. about 925 . Therefore, by taking this figure into account, the total estimated number of towns in the nation in 2020 will be raised double of 2007 figure. Compared with the data, the availability of towns whether it is large, medium and small along the routes that recorded as the maximum distance among all towns. It also records above the average distance gap $17 \mathrm{~km}$. It is mostly seen along the route of Assossa $(31 \mathrm{~km})$, and Bale Goba $(23.9 \mathrm{~km})$. It also shows that the areas were predominantly occupied by the small villages and rural areas of the nation. Thus, it has less probability for the formation of conurbation and the rate of urban sprawl in the future.

\section{Summary and conclusion}

The industry has various roles that provide for all walks of life. However, the special advantage of the industry is enabling the passengers to travel from place to place. In terms of the nature of metropolitan linkage, there are at 
least five major outlets that can link Addis Ababa with the hinterland. These are the Gojam, Nekemte, Jimma, Mojo and Dessie outlets that interlink the city with all outward and inward directions of the nation. The sector has also contributed a lot to the growth of towns. According to MoCUD, (2007) there are about 70 medium towns and 15 to 20 large towns. It is found that on average, there are more than 15 major towns that have made direct link with the center. Again, with regard to the presence of towns, the number of major and small towns found along the main outlets was about 42 . The largest number of towns was found along Inde Silassie, Gambel and Gonder, and it was 78, 53 and 52 respectively. Thus, this availability of towns comes mainly because of the route access and passenger transport.

It is also found that the average distance found between major towns and all towns was about $45 \mathrm{~km}$ and $17 \mathrm{~km}$ distance respectively. This shows that throughout these outlets that begin from origin to destination, the major towns would be located at every $45 \mathrm{~km}$ intervals. The distance gap between the major towns is very high along Gambela, Assossa, Gonder, Jinika and Assaita routes: $61,55,53,52$, and $52 \mathrm{~km}$ respectively. While, again, the lowest distance gap between the major towns which was below the average $(45 \mathrm{~km})$ is found along Jijiga $(30 \mathrm{~km})$, Inda Sillasie $(40 \mathrm{~km})$ and Bonga $(41 \mathrm{~km})$. It also indirectly connects the town and rural areas that are found at the two sides of the main routes. Commonly, it also connects the places that are located within 10 to $20 \mathrm{~km}$ and more radius of the town along the routes. Thus, it has high probability for the expansion of urban sprawl and formation of conurbation in the future.

It also has a key role in transitional towns where the passengers would stay on average for more than half an hour. During this stay of passengers in the town, they may consume the available material. It has significant benefit for the towns that are seen along the route and which can also serve as a rest place. It appears that LDB provides numerous roles to transitional towns. It is found that all towns that are found along the main route benefit a lot from the industry. LDB has a visible role in the selected transitional towns.

In general, the sector contributes to all walks of life, not only to intercity travel. It is described that the sector provides overwhelmingly to all beneficiaries. The prominent role that the sector contributes to the society is creating job opportunities for the jobless people. The estimated amount of associations and engaged individuals that is only within Mericato Bus in 2011 and 2012 were more than 30,000 individuals.

The general conclusion of this article is that the availability of towns comes mainly because of the routes access and road passenger transport. The presence of these routes contributes more to the rise of urbanization and it has fewer roles for the making of towns and rural areas found around certain radius at the two sides of the highways. The LDB along the line has contributed a lot to metropolitan linkage. The increment of the gap between towns results 
in the reduction of physical linkage of the city. All the geographic, political, economic and social aspects of the city would become more advantageous and contribute a lot for the metropolitan linkage with the hinterland. Therefore, the dispatch of buses has a key role for the major outlets and metropolitan linkage of the city and it enhances the personal and economic opportunities by creating job opportunities for the job seekers.

\section{Recommendations}

As to the foregoing discussion, the following key points were provided as possible suggestions to improve the overall situation of supply and demand of long distance bus transport. The following strategies were forwarded in priority order in terms of their weight for government and Associations.

1. Work with stakeholders to reduce the congestion of passengers in the terminals.

2. The association should serve the society $24 / 7$ or $18 / 7$.

3. Open and expand a ticketing office and develop a web site.

4. The government should expand Long Distance Bus Services to the neighboring countries.

5. Provide light food and soft drinks to customers in both outgoing and incoming travel.

6. The association should upgrade the standards of Mercato Bus Terminals.

7. Shift and decentralize the service sectors and selected International and Federal offices to the preferred Regional Capitals.

8. The government should upgrade the Associations into Companionship or Corporation.

9. Require proper and timely data handling at all levels of Transport Offices and the Associations.

\section{Acknowledgments}

The author would like to thank the staff at Federal Transport Authority of Ethiopia for providing various secondary data and willingness to provide primary information. Of them, his special thanks goes to $\mathrm{Mr}$ Animute Kassie for his data provision and GIS work and it also goes to Ms Meseret Hailu for encoding the data so it would become suitable for this presentation. All errors, particularly those related with the survey data, are the responsibility of the author. This article is designed based on a study that was sponsored by Arba Minch University research fund and UNISA bursary. 


\section{References}

Addis Ababa Chamber of Commerce (AACC), 2009. The Management of Commercial Road Transport in Ethiopia. Addis Ababa: Private Sector Development Hub/ Addis Ababa Chamber of Commerce and Sectoral Associations.

Addis Ababa City Government, office (AACGO) of the Mayor 2011/12.

Addis, Y., 2003. The Extent, Variations and Causes of Road Traffic Accidents in Bahir Dar. MA thesis. Addis Ababa University School of Graduate Studies, Addis Ababa.

Arowolo, O. O., 2010. Country Case Study: Ethiopia. Country case study prepared for the Center for Global Development Working Group on UNFPA's Leadership Transition. [pdf]. Available at: <http://www.cgdev.org/doc/Ethiopia-case-study.pdf $>$.

Bekalo, M. T., 2009. Spatial Metrics And Landsat Data For Urban Land use Change Detection. Dissertation submitted in partial fulfilment for the requirement for the Degree of Master of Science in Geospatial Technologies. The Department Of Information Systems, Universitat Jaume I, Castellon, Spain.

Belachew, M., 1997. Some Thoughts on Intra-Urban Transport Problems in Ethiopia The Case of the Anbessa City Bus Transport. Ethiopian Journal of Development Research, vol. 19 (1).

Central Statistical Agency of Ethiopia (CSA), 2007. Census (2007): preliminary. Available at: $<$ http://www.csa.gov.et/pdf/Cen2007_prelimineray.pdf.> [Accessed 7 December, 2008].

Central Statistical Agency of Ethiopia (CSA), 2007. Compilation of Economic Statistics in Ethiopia. Addis Ababa.

Central Statistical Authority of Ethiopia (CSA), 1984. Ethiopia 1984: Population and Housing Census of Ethiopia. Preliminary report. Addis Ababa.

Central Statistical Authority (CSA), 1996. The Federal Democratic Republic of Ethiopian Central Statistical Authority Transport and Communication Statistics Statistical bulletin No 246. Addis Ababa.

Central Statistical Authority of Ethiopia (CSA), 2009. The Federal Democratic Republic of Ethiopian Central Statistical Authority Annual Abstract Country Summary. Addis Ababa.

Cohen, B., 2004. Urban Growth in Developing Countries: A Review of Current Trends and a Caution Regarding Existing Forecasts. World Development, 32 (1), pp. 23-51.

Ethiopian Federal Transport Authority (EFTA), 2011. The Directorate of Commercial Passenger Quality Certificate Office. Addis Ababa.

Ethiopian Federal Transport Authority (EFTA), 2012. The Directorate of Commercial Passenger Quality Certificate Office, the survey of supply and demand from the main Bus terminal of Addis Ababa. Addis Ababa.

Ethiopia Road Authority (ERA), 2005. Ethiopian National Urban Transport Policy, Urban Transport Study and Preparation of Pilot Project of Addis Ababa. Final Report. December 2005, Addis Ababa. 
Gebeyehu, M., Takano, S., 2007. Diagnostic Evaluation of Public Transportation Mode Choice in Addis Ababa. Sapporo Japan Journal of Public Transportation, 10 (4), pp. 27-50.

Ministry of Finance and Economic Development (MoFED), 2006. Ethiopia: Status Report on the Brussels Pogramme of Action (BPoA) for Least Developed Countries (LDCs). January 2006, Addis Ababa.

Ofcansky, T. P., Berry, L. eds., 1991. Ethiopia: A Country Study. Washington: U.S. Library of Congress.

Tadesse, A., 2006. Road Freight Transport in Ethiopia with Special Emphasis on Addis Ababa-Djibouti Corridor. MA thesis. Addis Ababa University School of Graduate Studies, Addis Ababa.

\section{About Author}

\section{Fekadu KASSA}

He is lecturer at Arba Minch University, Department of Geography and Environmental Studies. He obtained a BA degree in Geography from Dilla University, and an MA degree in Regional and Urban Planning at the Department of Geography and Environmental Studies, Addis Ababa University. He is presently working on his $\mathrm{PhD}$ in transport geography within the Department of Geography in UNISA, which is supported by the Ethiopian Ministry of Education.

fkdu2003@gmail.com 